Théologiques

Théologiques

\title{
Nouveau regard sur les ritualités religieuses
}

\section{Denis Jeffrey}

Volume 4, numéro 1, mars 1996

Les Rites : céder en résistant

URI : https://id.erudit.org/iderudit/602434ar

DOI : https://doi.org/10.7202/602434ar

Aller au sommaire du numéro

Éditeur(s)

Faculté de théologie de l'Université de Montréal

ISSN

1188-7109 (imprimé)

1492-1413 (numérique)

Découvrir la revue

Citer cet article

Jeffrey, D. (1996). Nouveau regard sur les ritualités religieuses. Théologiques, 4(1), 95-110. https://doi.org/10.7202/602434ar

\section{Résumé de l'article}

Le fidèle qui allume un cierge et demande la satisfaction d'un souhait ou celui ou celle qui médite au son des vagues océaniques afin de trouver la paix intérieure pratique, selon le sens commun, un rituel religieux. Dans ce texte, nous proposons une compréhension du rituel qui tient compte de l'émancipation du sujet religieux, c'est-à-dire celui qui constitue sa religion personnelle à partir de ses propres expériences. Par conséquent, nous proposons un élargissement de la définition classique du rituel religieux en recentrant l'analyse sur les modalités fonctionnelle et symbolique de la ritualité de tous les jours. 


\title{
Nouveau regard sur les ritualités religieuses
}

\author{
Denis JEFFREY \\ Faculté des sciences de l'éducation \\ Université Laval
}

L'homme valorise à l'extrême certains instants rares, fugitifs et violents, de son expérience intime. Le Collège de Sociologie part de cette donnée et s'efforce de déceler les démarches équivalentes, au cœur même de l'existence sociale, dans les phénomènes élémentaires d'attraction et de répulsion qui la déterminent comme dans ses compositions les plus accusées et les plus significatives telles les églises, les armées, les confréries, les sociétés secrètes.

Roger CAILLOIS, Nouvelle Revue française, $1^{\text {er }}$ juillet 1938.

\section{RÉSUMÉ}

Le fidèle qui allume un cierge et demande la satisfaction d'un souhait ou celui ou celle qui médite au son des vagues océaniques afin de trouver la paix intérieure pratique, selon le sens commun, un rituel religieux. Dans ce texte, nous proposons une compréhension du rituel qui tient compte de l'émancipation du sujet religieux, c'est-à-dire celui qui constitue sa religion personnelle à partir de ses propres expériences. Par conséquent, nous proposons un élargissement de la définition classique du rituel religieux en recentrant l'analyse sur les modalités fonctionnelle et symbolique de la ritualité de tous les jours.

On entend très souvent, de la part des uns et des autres, que toute personne désire à la fois une vie sans histoire et une belle histoire de vie. Mais qu'est-ce qu'une vie sans histoire? Une vie sans problème ni émotions fortes, sans fracture ni désordre, sans faute ni dérèglement, vécue dans le confort que procurent les bonnes mours, les règles de conduite inébranlables et les morales rassurantes. Qu'advienne un événement 
déchirant, que surgisse l'angoisse ou la peur et tout devient menaçant; un univers ordonné pour l'éternité risque de basculer dans le chaos. Une vie sans histoire peut-elle être une belle histoire de vie? Toute histoire de vie n'est-elle pas tramée d'un bouquet d'émotions, de découvertes, de frissons, de désespoirs et de bonheurs? Une belle histoire de vie, en somme, qui serait un long fleuve tranquille, n'existe que dans la fiction romanesque. Une fiction bien morne, car la vie est tissée d'une diversité incroyable d'événements disparates et inattendus, le plus souvent sans conséquences graves, mais quelquefois irréparablement insupportables. Ce sont des événements qui apparaissent sans crier gare pour rompre l'écoulement régulier du temps.

Si on prête attention au babillage ambiant, on se rend compte de la duplicité des passions. Même ceux qui n'aiment pas s'éloigner du traintrain quotidien se laissent quelquefois entraîner dans une sorte de tourbillon qui brouille le cours familier des choses. On parlera d'une exaltation passagère qui brise la quiétude. Ce sont ces « instants rares » qui confèrent à la vie sa pleine valeur.

Certains ont l'esprit aventureux. Loin du péril, loin de l'intrigue, leur vie semble monotone. Ils se lancent dans des entreprises audacieuses : un projet financier, scientifique, amoureux; ils cherchent à relever des défis, à explorer des nouveaux mondes. Ils sont fascinés par l'inconnu, l'imprévisible, l'inédit, l'inexpliqué, la nouveauté. Ils aiment goûter la fécondité des changements et des bouleversements. Ils sont attirés par des expériences exaltantes ${ }^{1}$ qui procurent une sorte d'enchantement.

Cette attirance insatiable pour l'exotisme, pour l'épreuve, pour les aventures périlleuses, pour les situations extrêmes ou pour la violation des normes s'exprime par le rituel de transgression. La conduite transgressive est l'une des plus importantes expériences du sacré. On utilise habituellement la notion de transgression pour rendre compte de ce qui est vécu à l'approche de l'interdit.

À la frontière de ce qui est connu, ordonné, habituel, familier, profane, se profilent les principaux interdits. L'interdit n'est pas nécessairement posé par une instance dominante édictant la morale; devient aussi interdit ce qui excède une personne. L'interdit agit comme une limite ou un seuil et devient pleinement sacré lorsqu'il est actualisé ${ }^{2}$. Par exemple, le fait d'interdire à un enfant de manger tel aliment ne sacralise pas l'ali-

Voit David LEBReton, Passions du tisque. Paris, Métailié, 1991.

2 Voir Georges Bataille, L'érotisme. Paris, Minuit, 1957. 
ment pour autant. Il faut plutôt comprendre que peut être vécu par rapport à cet aliment un désir de transgression " ex-posant " l'interdit. L'enfant est confronté à un interdit dans la mesure où est convoqué ce qui l'excède. L'expérience de l'excès, le plus souvent vécu dans un sentiment de terreur fascinante ou de jouissance angoissée, caractérise, sous une forme paroxystique, l'expérience du sacré.

Nul ne pourrait vivre dans un monde stable et ordonné dans lequel on devrait uniquement respecter les interdits. Les interdits, de temps à autre, doivent être transgressés ${ }^{3}$. La fonction transgressive du rituel vise expressément à mettre en scène un interdit. S'approcher de l'interdit lors de conduites impliquant la dépense gratuite et le jeu risqué notamment procure une grande puissance de vie utile pour guérir une blessure existentielle, pour négocier un changement, pour créer, pour enchanter la vie, ou, ultimement, pour résister à la mort.

\section{Rituel, interdit, représentation symbolique}

Tout le monde n'est pas téméraire. La plupart suivent la routine. Cependant, lorsqu'un événement imprévu survient, on s'emploie le mieux qu'on peut à le négocier en utilisant ces outils hérités des traditions que sont le rituel et son symbolisme. En somme, qu'on soit aventurier ou pantouflard, on devra réagir aux diverses situations de la vie qui sont autant d'épreuves qui dynamisent les fonctions du rituel. Ce sont des moments forts auxquels chacun doit apprendre à faire face. Alors que certaines situations de moindre importance ne demandent que quelques ajustements mineurs, d'autres impliquent une modification majeure dans les orientations de vie. C'est là qu'intervient la capacité propre de chacun de ritualiser et de symboliser ce qui l'excède.

Tous les rituels présentent un caractère commun: ils convoquent des interdits. Les rituels sont les différentes conduites, individuelles et collectives, prescrites ou spontanées, domestiquées ou violentes, impliquant un rapport aux interdits. Il convient d'envisager l'étude du rituel, dans la société actuelle, à partir de la distinction entre le rituel institué et le rituel instituant. On connaît passablement bien le rituel institué. Il vise à actualiser périodiquement un mythe. Un rituel, selon la formule de Van der Leeuw, «est un mythe en action ${ }^{4}$ ". Le rituel institué présuppose le

3 Voir Roger CailloIs, L'homme et le sacré. Paris, Gallimard, 1970.

4 VAN der Leeuw, cité par Georges Gusdorf, Mythe et métaphysique. Paris, Flammarion, 1953, p. 23. Cette formule est reprise par un nombre important de spécialistes du religieux, notamment Roger Bastide qui dira " tout rituel est commé- 
mythe, il réfère à un système codifié de croyances, plutôt rigide, qui conditionne les pratiques de manipulation des interdits.

Parce qu'il prend le sens à la fois d'un acte conditionné et d'un effort pour maîtriser la négociation des interdits, le rituel institué, dans une certaine mesure, tend vers la sécularité. La sécularité est le plus souvent associée à une disparition du sacré. Or, il est bien difficile de croire, dans une société où pullulent les sectes et les mouvements religieux, à la disparition du sacré. Nous devons plutôt supposer que l'expérience du sacré prend des formes différentes à travers l'histoire. Si l'on est attentif aux déplacements des expériences du sacré, on dira qu'il y a sécularisation dans une religion singulière. Partant de ce fait, il est possible d'affirmer que certaines formes d'expérience du sacré se sécularisent alors que des nouvelles formes apparaissent.

Selon une hypothèse que nous privilégions, la sécularité résulte de la perte d'enchantement lors d'une expérience du sacré. L'enchantement, à bien des égards, n'est pas uniquement lié à l'émerveillement. Ce sentiment repose sur la capacité humaine de s'ouvrir à une expérience qui l'expulse du monde quotidien. Or, une expérience du sacré excessivement encadrée dilue l'indéterminé et l'imprévisible qui sont les conditions de son émergence. Le tituel institué, dans sa forme achevée et fortement formalisée, renvoie à la morale d'une société dont le dessein ultime est de fixer des règles de conduite personnelle, de réglementer la vie sociale et de proposer des compensations futures. Cependant, lorsque le rituel institué devient uniquement un code prescriptif, un code de morale, nous dirons alors qu'il perd sa puissance de médiation avec les interdits et qu'il ne provoque plus d'expérience du sacré. À l'évidence, cette hypothèse ne saurait à elle seule expliquer les processus de sécularisation. Cependant, elle est incontournable pour comprendre la religion dans les sociétés modernes.

Dans la mouvance de cette hypothèse, un rituel religieux par trop institué, qui ne serait qu'une borne morale, atteint un point de saturation, se sclérose, s'effrite et devient caduc. C'est dire que le rituel institué perpétue des structures pétrifiées. En revanche, d'anciens rituels seront transformés, d'autres verront le jour. $\grave{A}$ bien des égards, des pratiques rituelles inédites permettront de s'allier aux forces sacrées, de s'en approcher 
homéopathiquement, lors d'une expérience intime. Ces derniers sont des rituels instituants. Contrairement au rituel institue qui reconduit un mythe parfois privé de la puissance de ses symboles, le rituel instituant est à l'origine d'une réorganisation du mythe. Le rituel instituant génère désordre, déséquilibre, discontinuité, et force un système mythique clos à se renouveler, à se complexifier. Cependant, on peut observer que la plupart des conduites rituelles instituantes finissent par prendre des formes instituées.

Tout rituel, dans son dynamisme fonctionnel, remplit le double rôle de protection et d'enchantement. Lorsque la fonction de protection domine, l'expérience du sacré perd sa vitalité. On dira que les rituels sont devenus ennuyeux et qu'ils ne sont plus propices à la vie religieuse. Au contraire, lorsque la fonction d'enchantement domine, par exemple lors d'un événement fondateur ou d'un moment de renouvellement, il y a alors danger de perdre pied dans la griserie de l'extase. C'est pourquoi ces deux fonctions doivent trouver leur équilibre. On parlera d'un équilibre tensionnel pour évoquer le dynamisme de ces deux fonctions primordiales du rituel. Par conséquent, chaque organisation religieuse détient le mandat de veiller à cet équilibre.

Les notions de symbolisme ou de symbole renvoient aux représentations, aux images, aux productions de l'imaginaire qui désignent les interdits. Ainsi, un interdit est toujours exprimé, dans une culture donnée, sous la forme d'une image ou d'une représentation symbolique.

Les symboles ou représentations symboliques sont utilisés pour exprimer ce qui échappe à la maîtrise individuelle et collective. Le symbole est un produit de l'imagination visant à représenter une charge émotive très forte qui ne trouve pas de mots pour se dire. La mort, l'incompréhensible, l'inconnu, le mystère, l'obscène, le destin, l'étrangeté, le monstrueux, l'aléa, le refoulé sont des contenus paradigmatiques du symbole. 11 est rare qu'on puisse traduire dans une langue familière des sentiments très intenses de terreur ou de fascination. C'est que ces sentiments exposent à une irrémédiable perte de contrôle de soi. La symbolisation sert à communiquer ce qui se vit dans les parages de l'interdit et implique une mâ̂trise par la représentation.

La représentation symbolique se substitue à des sentiments intenses tels que la peur, la vengeance, la terreur, la panique, la rage, la colère, la fascination, l'éblouissement, etc. En fait, la représentation symbolique diminue le choc des impressions fortes et en permet l'expression. La maîtrise de soi, d'autrui et du monde ambiant dépend du jeu paradoxal des 
symboles. On peut s'imaginer, en l'absence d'un système symbolique adéquat et signifiant, qu'on soit saisi de panique.

$\grave{A}$ titre d'illustration, l'interdit du cannibalisme trouve difficilement de mots pour se dire. Même sous le couvert du sous-entendu, le cannibalisme soulève horreur et dédain. Pourtant, cet interdit apparaît sous diverses formes symboliques. À titre d'exemple, sa représentation symbolique renvoie, dans les religions judéo-chrétiennes, au vin et au pain transmués en sang et en corps de Jésus. L'interdit de l'inceste est symbolisé, dans la mythologie hellénique, dans le récit œdipien. La figure d'CEdipe demeure encore aujourd'hui le symbole premier pour évoquer les liens incestueux entre l'enfant et sa mère. En somme, l'interdit s'exprime par des symboles que les pratiques rituelles tendent à rendre manifestes.

Dans son sens premier, l'interdit est un «impossible à dire », un « impossible à concevoir "; c'est pourquoi nous devons référer à des images ou des symboles pour faire entrer l'interdit dans le champ des représentations. Les grands mythes religieux sont des mises en récit des interdits. Dans les sociétés traditionnelles, le rituel est strictement lié aux mythes. Le rituel représente le moment lors duquel le mythe est mis en scène.

Les interdits sont nombreux et traversent la multitude des relations qu'une personne entretient avec elle-même, autrui et son milieu de vie. Il est très difficile de repérer l'interdit derrière le symbole. L'on sait toutefois que l'expression d'un interdit implique de fortes charges émotives. $\grave{A}$ vrai dire, toute manifestation de violence - associée à une perte, une agression, une frustration, une rupture, un rite de passage, un trauma, une conduite risquée, un décès, une guerre, un événement brutal - signale un ou des interdits.

\section{Rituel et mythe}

Mircea Eliade a proposé une définition du mythe qui retiendra notre attention ${ }^{5}$. Pour l'historien des religions, le mythe est un récit des origines, une histoire avant l'histoire, in illo tempore, qui a pour fonction d'instaurer l'ordonnancement du monde. Le mythe raconte l'événement fondateur et l'ordre qui en découle. Dans toutes les sociétés traditionnelles, les mythes sont intimement liés aux rites. En fait, selon une définition convenue en sciences religieuses, le mythe se constitue à partir d'interdits que les rituels évoquent dans certaines circonstances.

5 Mircea ElIADE, La nostalgie des origines. Paris, Gallimard, 1971. 
La plupart des mythes racontent qu'une transgression a éloigné l'humain d'un lieu par la suite devenu interdit. L'histoire des mortels découle, selon la logique mythique, d'une transgression originaire. À la suite de celle-ci, il est interdit aux hommes de revenir en arrière, in illo tempore. Son sort se résoud en un difficile éloignement de l'âge d'or primordial.

Toutefois, le rituel conforte l'être humain par une mise en scène des interdits le séparant de ce pays lointain dans lequel les dieux et déesses ont élu domicile. L'acte originaire de la transgression est répété symboliquement lors de certains moments privilégiés. Grâce à des pratiques rituelles appropriées, le mortel retrouve, pour un laps de temps déterminé et sous un mode symbolique, le souvenir heureux de son immortalité.

On ne pourrait uniquement vivre dans un monde où est interdit l'accès à l'immortalité: on doit croire à la possibilité de transgresser les interdits et ainsi accéder, du moins symboliquement, à l'immortalité. C'est la gageure conditionnelle à la vie humaine. Or, cette transgression menace l'ordonnancement du monde; c'est pourquoi elle doit être soumise à des procédures rituelles très strictes.

C'est dans un perpétuel décalage entre le présent et le rêve de retrouver l'origine, la souffrance de la mortalité et l'extase paroxystique ouvrant sur l'immortalité, l'incompréhensible dont la mort est le paradigme et le mythe vécu, que se constitue la ritualité. La ritualité protège de la souffrance de la mortalité car elle permet un accès symbolique à l'immortalité.

Il est facile de concevoir que l'interdit qui délimite le monde divin du monde humain doit être transgressé, et que c'est là le but de toute religion de veiller à cette transgression. Or, rappelons que la transgression n'abolit pas l'interdit mais le confirme. Par la transgression, l'humain éprouve ses limites, constitue sa structure identitaire et conjure l'angoisse de sa mortalité.

On comprend maintenant pourquoi l'extase rituelle procure une énergie de vie pleine de satisfactions. Inversement, la perte de l'accès à l'immortalité est mortifère. Ainsi, l'accès à l'immortalité - par l'intermédiaire de la croyance en l'utopie du paradis perdu ou celle d'une idéologie faisant la promotion des lendemains qui chantent, par l'acceptation tragique du présent ou par l'assomption de la filiation -, est conditionnelle à l'enchantement de la vie.

Dans un sens anthropologique, on peut comprendre la ritualisation des interdits comme une maîtrise du temps qui passe et de l'angoisse suscitée par la mort. Annick Barrau, à la suite de Louis Vincent Thomas, 
souligne que "de toutes les violences, la mort est sans conteste la plus puissante car la plus destructrice, la plus désorganisatrice mais aussi la plus fécondante, la plus fondatrice ${ }^{6} \%$. Il n'y a pas de retour apaisant vers la source originaire d'où provient l'humain. Les sagesses religieuses nous ont appris qu'il n'est ni facile d'apprendre à faire le deuil du paradis perdu, ni facile de se préparer pour le grand saut dans l'au-delà inconnu. Les passages de l'immortalité à la mortalité, puis de la vie à la mort, constituent les frontières les plus éprouvantes à traverser. C'est la raison pour laquelle chaque grande religion articule son récit mythique autour de ces thèmes fondamentaux.

\section{Le petit récit}

Il n'est certes pas possible d'aborder la notion d'interdit sans retourner aux traditions religieuses qui l'ont mythifiée. Cependant, l'incrédulité à l'égard des grands récits religieux oblige à poser autrement la question de la régulation des interdits. Même si les interdits sont libérés de leur mise en scène traditionnelle par un rituel institué, d'autres mises en scènes assurent les fonctions rituelles. On peut supposer que les grands récits religieux sont remplacés par des petits récits personnels ou collectifs se proposant d'exprimer l'événement instaurateur de leur organisation. Plusieurs moments forts de la vie tels la maladie, la perte d'un être cher, un accident, la crise amoureuse se constituent comme une rupture instauratrice d'un nouvel ordre personnel, et tiennent lieu d'événements fondateurs. Sur cette rupture, plusieurs construisent leur propre église en pigeant ici et là, dans les grands récits religieux anciens et nouveaux, certaines croyances, certains symboles, certains mythèmes, qui sont porteurs d'interdit, et qui vont motiver différents types de ritualisation.

Le mythe personnel est l'histoire qu'on raconte à ses proches, une histoire qui prend corps en marquant les caractéristiques identitaires. En somme, une personne (ou une communauté de personnes), en se racontant, en racontant les événements marquants de son chemin de vie, constitue son petit récit religieux, c'est-à-dire ses propres frontières identitaires que bordent les interdits. Le seul fait de raconter son histoire personnelle initie à soi, à sa propre subjectivité.

$\mathrm{Ce}$ qu'une personne raconte, en fait, ce sont les moments vécus intensément qui l'ont troublée, bouleversée, angoissée, exaltée, fascinée,

6 Annick BarraU, Mort à jouer, mort à déjouer. Socio-anthropologie du mal de mort. (Sociologie d'aujourd'hui), Paris, PUF, 1994, p. 39. 
fragilisée. Elle raconte comment elle a négocié une épreuve, une expérience difficile, un fait imprévu, la rencontre de l'inconnu, un malaise, une souffrance. En se racontant, elle fait advenir sa propre position de sujet de son récit, de sa parole, à la condition nécessaire qu'on veuille bien reconnaître cette parole. Une personne naît, en se racontant à travers autrui qui lui restitue sa parole, à sa propre vérité qui devient mythe. Ce mythe bien réel fonde la structure identitaire sur laquelle se construit son cheminement.

Plus d'une personne bricole son petit récit religieux ${ }^{7}$ en puisant dans la forêt des symboles de diverses traditions religieuses. Elle les assemble sous une forme cohérente et transmissible. Son univers symbolique se compose du croisement de ce qu'elle considère comme important et signifiant. Elle ose interpréter de son propre chef des événements marquants et des questions fondamentales en les reformulant avec des mots et des images personnelles.

Les rituels qu'elle pratique, individuellement et collectivement, sont intimement associés à ses constituantes identitaires. Si elle ne se reconnaît plus comme chrétienne, musulmane ou bouddhiste, il n'en demeure pas moins que la dimension religieuse reste prépondérante dans la définition de son récit identitaire même si elle ne sait plus la nommer. À la question: "êtes-vous chrétien?", elle répond " oui et non ", "peut-être ", " je ne suis pas sûr ", " pas complètement", "non si vous pensez que je crois en ceci ou en cela", "oui si vous me permettez de me situer par rapport à la position du pape en ce qui concerne... ».

Certains revendiqueront l'appellation «Nouvel Âge " pour nommer une appartenance religieuse qui ne correspond pourtant pas tout à fait à ce qui est vécu. C'est une appellation par défaut. D'autres diront " je ne suis plus religieux mais je crois à la spiritualité ". Cette formulation porte le désir de participer à une vie religieuse hors du cadre des institutions. Comment nommer ce qui est le plus souvent éprouvé dans la solitude de la conscience? Quels sont les mots qui décrivent le vécu religieux d'une personne du $\mathrm{XX}^{\mathrm{e}}$ siècle? Comment peut-elle assumer le statut de chrétien, par exemple, alors qu'elle ne supporte plus le clergé et les positions doctrinales de l'Église? Pourra-t-elle être en partie chrétienne, en partie raëllienne, en partie bouddhiste, en partie musulmane? Ce syncrétisme des croyances trouve difficilement de mots pour se dire.

7 Voir Raymond LEMIEUX et Micheline MILOT, dir. Les croyances au Québec, Esquisses pour une approche empirique. Les cahiers de recherche en sciences de la religion, Vol. 11, 1992. 
Le petit récit du "sujet postmoderne ${ }^{8}$ " n'a pas à être véridique, ce n'est pas un récit "scientifique». Cependant, il doit être reconnu pour devenir la vérité du sujet. Ce dernier n'arrive pas en une seule fois à se dire, à formuler son histoire de vie, à dire sa vérité. Il recommence chaque fois qu'il se rencontre au détour d'une écoute attentive. Le sujet se raconte, s'écoute, écoute autrui se raconter pour enrichir son histoire. La vétité n'est jamais facile à dire ni à voir, les médiations sont nombreuses qui rendent propice l'advenu de la vérité. Chaque expérience de vie comme chaque rencontre permet au sujet de continuer son cheminement dans sa vérité. Vérité, en fin de compte, qui est son cheminement.

Faire l'expérience de sa vie, c'est quelquefois s'essayer autrement, s'exercer à être libre, chercher l'occasion d'une situation inédite pour s'expérimenter. Le sujet qui se raconte jouit d'une distance, d'une brisure critique et émancipatrice, mais parfois également angoissante. Cette distance à soi, cet espace de jeu, est une fenêtre ouverte sur lui-même, autrui et son milieu de vie.

\section{Fonction «pontificale » du rituel}

Le rituel est "pontifical " en ce qu'il protège contre la violence d'une forte charge émotive et permet d'y puiser des énergies nouvelles. Le mot pontifex signifiait, à l'époque romaine archaïque, faiseur de ponts. Alors qu'autrefois, seul le chef des prêtres, le pontife, pouvait se servir d'une puissance et d'un symbolisme sacrés pour ériger un pont reliant deux rives que les dieux avaient séparées, il semble aujourd'hui que chacun s'autorise à construire ce pont, par lui-même ou avec l'aide d'autrui. Cette fonction du rituel évoque la capacité propre à chacun de négocier un moment fort de la vie, en construisant des ponts sur l'angoisse qui coule en lui.

Sont donc pontificales ces conduites qui aident à traverser un temps fort de la vie. On ne doit pas cacher que la vie n'est pas un long deuil tranquille. Chacun est, plus souvent qu'il ne le pense, assailli par des petits ou des grands événements qui l'expulsent hors des lieux convenus. Un changement de carrière, la confrontation avec l'étranger ou l'étrangeté, une fissure dans les croyances, le passage à la vie adulte, forcera à transiter, à pontifier, à ritualiser. Chacune des secousses existentielles, qui sont le plus souvent vécues comme une sorte de sentiment de violence intime - on peut se faire violence ou être introduit à la violence - , trouvera à s'apaiser par un processus de ritualisation. 
Qu'on pense à l'importance de l'anniversaire de naissance. C'est pour pontifier la violence irréparable du temps que chacun aime se retrouver avec les siens pour vivre les transitions de l'âge. On se réunit aurour d'un bon repas pour célébrer une personne estimée. Puisque "c'est à son tour de se laisser parler d'amour ${ }^{9}$ ", on invoque les meilleurs souvenirs, on offre des cadeaux, on prépare un gâteau d'anniversaire parsemé de petites bougies symbolisant le feu sacré de la vie et le temps qui passe. On demande à la personne fêtée de formuler un vœux secret avant de souffler sur les chandelles qu'elle doit éteindre d'un seul souffle. La magie du rituel et de son symbolisme aide à accepter l'action du temps et réconforte les attachements que le jubilant entretient avec ses proches.

À côté de ces petits moments d'ivresse passagère que sont ces fêtes tranquilles d'anniversaire de naissance assurant la transition d'une étape de la vie, d'autres fêtes soulignant un passage semblent plus critiques. Il y a des tournants décisifs dans l'histoire de vie d'une personne qui impliquent une plus grande puissance de vie. Qui n'a pas entendu cette histoire d'une fête anniversaire qui a durée toute la nuit lors de laquelle drogues, alcools et sexes circulaient librement. Certaines célébrations qui marquent la fin d'une épreuve, par exemple la fin des études universitaires, sont l'occasion d'une ritualisation. Certains vont mettre beaucoup d'énergie pour organiser une sorte de petite bacchanale qui marquera à jamais la mémoire. Plusieurs moments de transition exigent une grandiose dépense d'énergie. On rapporte quelquefois dans les journaux ces histoires au sujet d'un enterrement de vie de garçon qui se termine mal. Ces débauches amicales sont propices à la manifestation des tensions affectives vécues à l'intérieur du groupe d'amis.

On reconnaît assez facilement le bien fondé de ritualiser un simple anniversaire de naissance ou de ritualiser l'entrée au collège ou à l'université. On reconnaît également la nécessité de ritualiser la naissance d'un enfant, l'union de deux personnes ou le décès d'une personne chère. Mais on oublie que tous les moments importants de la vie demandent un rituel approprié. Le passage à la vie adulte, les premières menstruations, le mariage, le divorce, la maladie, la rencontre d'une nouvelle personne, les changements de saison nécessitent une ritualité particulière.

La plupart des rituels reconduisent un désir pontifical. Parce que nous sommes des êtres inachevés, nous devons sans cesse nous adapter -

9 Thème d'une chanson populaire de l'auteur-compositeur-interprète Gilles VIGNEAULT, tiré de "Gens du pays ". Les Québécois chantent cet air-ballade à ta personne fêtée. 
c'est-à-dire pontifier nos transitions : attacher ensemble, avec cohérence, chacun des moments de la vie - pour répondre à la fois à nos besoins primaires et aux demandes du milieu de vie. On sait qu'il n'est pas facile de pontifier les moments forts de l'existence. Lorsque la chaîne reliant à soimême, à autrui ou à son milieu de vie est rompue ou doit être rompue et réaménagée, cela provoque un sentiment de dissolution ou d'abandon. C'est le plus souvent en cherchant un réconfort auprès des siens qu'on arrive à apaiser des sentiments extrêmes. Des événements forts tels la rupture amoureuse, le décès, la perte, la maladie, la guerre, provoqueront un sentiment de dissolution beaucoup plus fort et pourront engendrer un grand désordre intérieur et même un état de crise.

À travers une expérience de crise, toutes les énergies de vie entrent en jeu pour rétablir un bris du continuum existentiel. Précisons que la crise est une réaction normale des mécanismes de régulation de la vie ${ }^{10}$. Elle indique un changement qui pourra être bénéfique dans la mesure où il permet d'éviter une crise plus violente. La crise n'est pas une défaillance mais peut le devenir lorsqu'elle n'arrive pas à se ritualiser et à se symboliser. Le rituel, en fait, comprend des fonctions préventives et thérapeutiques. La ritualisation d'une brisure du cours régulier de la vie permettra peut-être d'éviter une crise incontrôlable.

\section{Rituel et fusion collective}

On évoque souvent ces grands rituels de communion collective que sont les rassemblements religieux et politiques, et même les concerts rock. Mais on accorde une moins grande importance à tous ces rituels de communion que sont les différents festivals de musique, de cinéma, de bleuets ou de homards; ou à ces rencontres folkloriques que sont les épluchettes de blé d'Inde, les journées à la cabane à sucre ou les rassemblements lors d'un événement sportif. Des gens se réunissent dans des enceintes consacrées pour éprouver des sentiments communs.

Lors de la fin de la télésérie américaine Cheer's, une grande foule s'est assemblée dans un amphithéâtre de Boston pour vivre ensemble les dernières minutes de l'émission. Il y a dix ans, lors de la dernière de la télésérie MASH, plusieurs groupes se sont réunis chez les uns et les autres pour ritualiser la disparition à l'écran de personnages que tous avaient appris à connaître et à aimer. Alors que l'équipe de hockey les

10 Voir René KAËS et al., Crise, rupture et dépassement. Analyse transitionnelle en psychanalyse individuelle et groupale. Paris, Dunod, 1979. 
Canadiens de Montréal jouait à Los Angeles lors des éliminatoires de la coupe Stanley au printemps 1993, un groupe important de supporters ont préféré payer pour suivre le match sur écran géant au Forum de Montréal plutôt que de rester à la maison, comme c'est la coutume, pour suivre la retransmission à la télévision.

Baigner dans l'ambiance fusionnelle de la foule permet à chacun de vivre une expérience féconde de solidarité harmonieuse. Le rituel initie chaque personne singulière dans le destin commun de tous les êtres humains. Les rassemblements ponctuels offrent la possibilité de partager et d'exprimer des émotions difficiles à vivre seul. Ce sont des rituels qui interpellent la fonction de communion assurant la reliance ou l'êtreensemble ${ }^{11}$.

Lors d'une crise existentielle - sur arrière-fond de crise de civilisation, devrait-on ajouter - certains s'en remettront aux rituels traditionnels institués tels le rituel chrétien de la confession, le rituel de la cure psychanalytique ou tous ces rituels qui appartiennent au champ des médecines douces; d'autres vont plutôt s'adonner à des conduites que nous n'avons pas appris à classer parmi les rituels religieux. Ce sera une expédition périlleuse, une "expérience de marge" (itinérance, alcoolisme, abus de drogue, prostitution, etc.), une dépense gratuite ou ce qu'on a l'habitude d'appeler une folie passagère - par exemple un investissement à la bourse ou un changement de style de vie. Toutes ces conduites sont autant de rituels religieux qui échappent aux analyses traditionnelles. Pourtant, ce qui est vécu à l'égard des interdits s'impose comme l'une des modalités les plus importantes dans les rapports à soi, à autrui et à son milieu de vie.

\section{Les rituels de la vie de tous les jours}

Il est opportun de repérer les ritualités personnelles, celles auxquelles on n'accorde pas tout l'intérêt qu'elles méritent car on les considère comme insignifiantes. Ces ritualités personnelles jouent un rôle primordial dans le diagnostic que nous pouvons établir sur la santé d'un individu et d'une société. L'étude des ritualités du quotidien vise à faire apparaître le sens et la portée de ces pratiques dont le but lointain est de négocier la mortalité humaine afin d'enchanter la vie.

11 Voir Michel M AfFESOLI, Au creux des apparances. Pour une éthique de l'esthétique. Paris, Plon, 1990. 
Les définitions traditionnelles du rituel sont inadéquates pour saisir la diversité des nouvelles formes de rituel dans la société assujettie aux logiques de la modernité ${ }^{12}$. Il semble plus intéressant de faire ressortir la part de ritualité "instituante", c'est-à-dire nouvelle, dans la plupart des conduites quotidiennes. L'approche du rituel préconisée ici a comme tâche de signaler les fonctions rituelles dans les diverses activités de tous les jours. En d'autres mots, il s'agit de rendre compréhensible des ensembles de conduites individuelles et sociales, pour en faire ressortir un sens religieux non encore apparu. Ce point de vue n'enlève rien à toutes les théories qui abondent pour expliquer les conduites humaines. Cette perspective, qui s'inspire de la thèse des déplacements de l'expérience du sacré ${ }^{13}$, se veut complémentaire aux efforts visant à comprendre une personne dans ses multiples relations.

Les ritualités du quotidien prennent des figures multiples. À vrai dire, on peut faire ressortir pour chaque personne, dans la diversité de ses activités journalières, la part de ritualité que celles-ci contiennent. Peut-être apparaîtront-elles perverses, vicieuses et immorales, quelquefois violentes et parfois pacifiques, mais il faut considérer que la plupart des conduites rituelles instituantes concernent des personnes en rupture, qui éprouvent plus que d'autres la nécessité de mettre en acte leurs problématiques internes.

Le rituel ne se pratique pas uniquement lors de situations paroxystiques. La vie quotidienne est largement révélatrice de la présence manifeste ou latente d'expériences particulières à l'égard d'un interdit. Un bonjour anodin relève du rituel pontifical qui consiste à se lier, dans le mesure où il est possible de se délier. C'est là l'exacte définition du dynamisme du rituel.

Le simple bonjour, en effet, est une liaison fortuite qui permet à la fois de témoigner de la continuité d'un attachement et de la discontinuité du détachement. Le bonjour est une parole de transition permettant de pontifier nos liens avec autrui. Imaginons cette situation familière lors de laquelle, un matin, sur le lieu du travail, un collègue passe sans dire bonjour. Il n'y a là rien d'extraordinaire sinon que cela provoque un drôle de sentiment. Il y a, dans la parole absente, quelque chose qui chagrine, qui étonne, qui questionne, qui frustre, qui sonne le glas à l'habituel. En fait,

12 Voir Georges BalANDier, Le détour. Paris, Fayard, 1985.

13 Voir Yvon DESROSIERS, dir. Religion et culture au Québec. Figures contemporaines du sacté. Montréal, Fides, 1986. 
cette personne devient soudainement étrangère, inconnue, mystérieuse, différente. On cherchera à calmer notre malaise en s'informant, auprès des autres, de la situation inhabituelle créée par l'absence du bonjour matinal. On se demandera si on a pu, à notre insu, choquer cette personne. Il y a en effet un petit quelque chose de désorientant lorsque l'expression des liens quotidiens fait défaut. Ce sentiment de quelque chose d'étrangement inquiétant est causé par une défaillance du rituel magnifiquement domestiqué servant au maintien de nos relations quotidiennes.

Les diverses relations que chacun entretient avec autrui font partie d'une chaîne de ritualités si bien codées, si bien définies qu'il est facile d'oublier qu'elles sont très fragiles. Chacune est à la fois différente et semblable à toutes les autres. Sans le rituel, une personne aurait chaque fois à affronter cette différence dans un grand sentiment de frayeur et de fascination mêlées. La psychanalyse a mis en lumière les passions d'amour et de haine entre enfants et parents. On sait qu'existent des ritualités conditionnelles aux rapports éthiques entre les hommes et les femmes qui renvoient à ces passions originaires. Sans les mises en scènes quotidiennes qui introduisent les unes aux autres, on serait exposé à des sentiments de peur et d'angoisse qui viendraient envenimés l'existence.

Il est indéniable que nous devenons sensibles à la profondeur des ritualités qui assurent la continuité de la vie lorsque se profilent une rupture, une crise ou une discontinuité dans le cours régulier de la vie. Les crises individuelles et sociales se succèdent à un rythme effréné dans les sociétés modernes. Il est urgent d'observer les diverses mises en scène rituelles qui permettent à un individu comme à une société d'éviter l'accroissement de la crise. S'il y a un optimisme dans notre réflexion, c'est celui de croire que, dans la mesure où une crise est ritualisée, elle est un moment de changement et de renouvellement créateurs.

\section{Conclusion : nouveau regard sur l'expérience du sacré}

Dans la société moderne, les conduites religieuses semblent en manque de mots pour se dire, se confirmer et se faire reconnaître. On peut supposer que cela est dû au fait que les conceptions orthodoxes de la religion ne conviennent plus à l'image que se font nos contemporains d'eux-mêmes. Les attitudes religieuses, en effet, évoquent une époque rétrograde en rupture avec les formes avancées de la science et de la technologie. La méfiance à l'égard de la religion suppose également un reproche adressé aux autorités cléricales. On résiste au pouvoir des Églises qui, dit-on, exigent l'obéissance passive et totale. Au lieu de combattre les structures instituées des Églises ou de participer au débat qui les 
dynamise, plusieurs se sont retirés dans une religion personnelle qu'ils construisent avec les matériaux religieux qu'ils glanent ici et là au hasard des rencontres et des lectures.

Il est certain qu'on ne peut analyser les contingences de la religion personnelle de bon nombre de nos contemporains avec une conception de la religion qui se veut universelle. La religion personnelle est essentiellement relative aux "expériences du sacré " d'un sujet singulier. En revanche, son expérience retient l'attention dans la mesure où elle indique une posture subjective qui se rapporte à la détermination commune à tous les êtres humains. L'expérience fondamentale sous-jacente à la ritualité religieuse, qu'il est possible de décrire et d'évaluer de façon critique, repose sur l'hypothèse selon laquelle le rituel vise à négocier les interdits qui se présentent le plus souvent sous une forme transcendante servant à les exprimer. On ne doit certes pas oublier que l'interdit, lors d'un rituel instituant, cherche sa représentation symbolique.

La religion traditionnelle subit une mutation profonde. À l'évidence, elle est irréductible aux catégories proposées par les théologies monothéistes. Les mass media, les voyages, les déplacements de population vers les villes, l'immigration ont transformé radicalement l'idée que bon nombre de personnes se faisaient de la religion. Dans les grandes métropoles comme Montréal, New-York et Paris, il est possible de côtoyer toutes les cultures et sous-cultures religieuses. L'impérialisme d'une religion instituée s'est diffracté en une sorte de "polythéisme postmoderne" qui inspire la sensibilité religieuse de plusieurs personnes. L'importance de l'effritement d'un ensemble constitué de croyances partagées par tous en une multitude de pôles religieux reste encore à analyser. Cependant, on peut, sans trop se tromper, affirmer que la religiosité postmoderne favorise l'émancipation d'un sujet qui recompose son univers religieux d'une façon bien personnelle.

Pour comprendre les mécanismes de construction de la religion personnelle, il faut redéfinir la spécificité et les fonctions du rituel et de l'interdit, accepter d'analyser le remplacement des grands récits par des petits récits, examiner les nouvelles formes de croyances, étudier les expériences du sacré en retrait des monopoles religieux rigides et dogmatiques. Les problèmes ainsi posés sont simplifiés, mais ils restituent tout de même l'enjeu des disciplines qui prennent la religion personnelle pour objet. 